\title{
NOTE ON THE ITERATION OF FUNCTIONS OF ONE VARIABLE*
}

\author{
BY MORGAN WARD
}

1. Introduction. Let $E(x)$ be a real-valued function of the real variable $x$ for some specified range, and let

$$
E_{0}(x)=x, E_{1}(x)=E(x), \cdots, E_{n+1}(x)=E\left(E_{n}(x)\right), \cdots
$$

represent its successive iterations. The interpolation problem of defining $E_{n}(x)$ for non-integral values of $n$ was discussed some time ago by A. A. Bennett, $\dagger$ who reduced it formally to the solution of the functional equation

$$
\psi(x+1)=E(\psi(x)) .
$$

For if $\psi(x)$ satisfies (1) and if $n$ is any positive integer,

$$
\psi(x+n)=E_{n}(\psi(x)) .
$$

Hence on writing $\psi^{-1}(x)$ for $x$, where $\psi^{-1}(x)$ denotes an inverse of the function $\psi(x)$, we obtain the formula

$$
E_{n}(x)=\psi\left(\psi^{-1}(x)+n\right),
$$

defining $E_{n}(x)$ for a continuous range of values of $n$.

In this note, I propose to give an entirely elementary explicit solution to this problem of interpolation for all functions $E(x)$ subject to the following three conditions: $\ddagger$

(a). $E(x)$ is a real, continuous, single-valued function of the real variable $x$ in the range $a \leqq x<\infty$.

(b). $E(x)>x$ for all $x \geqq a$.

(c). $E\left(x^{\prime}\right)>E(x)$ if $x^{\prime}>x \geqq a$.

We may remark that the functional equation (1) is merely another form of a famous equation studied by Abel, $\S$

* Presented to the Society, June 20, 1934.

$\dagger$ In two papers in the Annals of Mathematics, (2), vol. 17 (1915-16), pp. 74-75 and pp. 23-60. This second paper contains references to the earlier literature. A. Korkine (Bulletin des Sciences Mathématiques, (2), vol. 6 (1882), pp. 228-242) seems to have been the first to consider this problem.

$¥$ These conditions are all satisfied by $E(x)=e^{x}$, the particular case discussed by Bennett in the first paper cited.

§ Works, vol. II, Posthumous Papers, 1881, pp. 36-39. 


$$
\phi(x)+1=\phi(f(x))
$$

as Abel himself showed.* Here $f(x)$ is a given function, and $\phi(x)$ is to be determined. This equation has been extensively investigated of late by modern function-theoretic methods. $\dagger$

2. A Simplification. As a preliminary simplification, we may assume that the constant $a$ in condition (a) is zero, and that $E(0)=1$. For if $E(a) \neq 0$, the function $E^{\prime}(x)=E^{2}(x+a) / E^{2}(a)$ satisfies conditions (a), (b), (c) with $a=0$, while $E^{\prime}(0)=1$, and $E(x)= \pm E(a)\left(E^{\prime}(x-a)\right)^{1 / 2}$. On the other hand, if $E(a)=0$, then $E_{2}(a)=E(0)>0$ by (b). Hence $E^{\prime \prime}(x)=E_{2}(x+a) / E_{2}(a)$ will satisfy (a), (b), (c) with $a=0, E^{\prime \prime}(0)=1$. Since $E(x)$ is continuous and monotonic increasing, it has a unique inverse $E_{-1}(x)$. Thus, if $E^{\prime \prime}(x)$ is given, $E(x)=E_{-1}\left(E_{2}(a) E^{\prime \prime}(x-a)\right)$.

From (b) and (c), it follows that for any positive integer $n$, $E_{n}\left(x^{\prime}\right)>E_{n}(x)$ if $x^{\prime}>x$. Since $E_{n}(x)$ is furthermore continuous by (a), it has a unique inverse which we shall denote by $E_{-n}(x)$. If we write $y=E_{n}(x)$, then by (b), $y \geqq E_{n}(0)$, so that $E_{-n}(x)$ is defined only for $x \geqq E_{n}(0)$. It is easily verified, however, that for any $x \geqq 0$,

$$
E_{n}\left(E_{m}(x)\right)=E_{n+m}(x)
$$

for all integral values of $n$ and $m$, positive or negative, for which the functions are defined.

3. Solution of (1). We shall next give a solution of the functional equation (1). Let $[x]$ denote as usual the greatest integer in $x$ so that

$$
0=E_{0}(0) \leqq x-[x]<E_{1}(0)=1 .
$$

Then

$$
\psi(x)=E_{[x]}(x-[x])
$$

is a monotonic increasing continuous solution of (1). For

$$
\begin{aligned}
\psi(x+1) & =E_{[x+1]}(x+1-[x+1])=E_{[x]+1}(x-[x]) \\
& =E\left(E_{[x]}(x-[x])=E(\psi(x)),\right.
\end{aligned}
$$

* Write (1) in the form $x+1=\psi^{-1}(E(\psi(x)))$. Then on substituting $\psi^{-1}(x)$ for $x$, we obtain $\psi^{-1}(x)+1=\psi^{-1}(E(x))$.

† See, for example, Picard, Lesons sur Quelques Equations Fonctionnelles, 1928, Chapter 4. For more recent papers, see the Zentralblatt für Mathematik under the index Funktionentheorie: Iterationen. 
and if $x^{\prime} \geqq x+1$,

$$
\begin{aligned}
\psi\left(x^{\prime}\right) & =E_{\left[x^{\prime}\right]}\left(x^{\prime}-\left[x^{\prime}\right]\right) \geqq E_{\left[x^{\prime}\right]}(0) \\
& =E_{\left[x^{\prime}\right]-1}(1) \geqq E_{[x]}(1)>E_{[x]}(x-[x])=\psi(x),
\end{aligned}
$$

while if $x+1>x^{\prime}>x$,

$$
\begin{aligned}
\psi\left(x^{\prime}\right) & =E_{\left[x^{\prime}\right]}\left(x^{\prime}-\left[x^{\prime}\right]\right)=E_{[x]}\left(x^{\prime}-[x]\right) \\
& >E_{[x]}(x-[x])=\psi(x) .
\end{aligned}
$$

The continuity of $\psi(x)$ is obvious if $x$ is not an integer $n$. Also if $x=n, \epsilon>0$, it is clear that $\lim _{\epsilon \rightarrow 0} \psi(n+\epsilon)=\psi(n)$. On setting $x=n-\epsilon, \epsilon>0$, we have $\lim _{\epsilon \rightarrow 0} \psi(n-\epsilon)=\lim _{\epsilon \rightarrow 0} E_{n-1}(1-\epsilon)$ $=E_{n-1}(1)=E_{n}(0)=\psi(n)$.

It follows that $\psi(x)$ has a unique inverse $\psi^{-1}(x)$. To determine it, let $x$ be given, and let the positive integer $k$ be determined by the inequality

$$
E_{k}(0) \leqq x<E_{k}(1)
$$

Then

$$
\psi^{-1}(x)=E_{-k}(x)+k .
$$

For first of all, $\psi^{-1}(x)$ is defined and continuous for all $x \geqq 0$. Secondly, from $(7), 0 \leqq E_{-k}(x)<1$ so that $k=\left[\psi^{-1}(x)\right]$, the greatest integer in $\psi^{-1}(x)$. Therefore

$$
\psi\left(\psi^{-1}(x)\right)=E_{k}\left(\psi^{-1}(x)-k\right)=E_{k}\left(E_{-k}(x)\right)=E_{0}(x)=x .
$$

Thirdly, since $E_{[x]}(0) \leqq \psi(x)<E_{[x]}(1)$,

$$
\begin{aligned}
\psi^{-1}(\psi(x)) & =E_{-[x]}(\psi(x)+[x])+[x] \\
& =E_{-[x]}\left(E_{[x]}(x-[x])\right)+[x]=x .
\end{aligned}
$$

We obtain then, on substituting in (3), the final result of this note:

$$
\begin{aligned}
E_{n}(x) & =E_{\left[n+k+E_{-k}(x)\right]}\left(n+k+E_{-k}(x)-\left[n+k+E_{-k}(x)\right]\right) \\
& =E_{\left[n+k+E_{-k}(x)\right]}\left(n+E_{-k}(x)-\left[n+E_{-k}(x)\right]\right) .
\end{aligned}
$$

Here the integer $k$ is determined by the inequality (7) and the formula is valid for all real values of $n \geqq 0$. The equation (5) may now be shown to hold for non-integral values of $m$ and $n$.

California Institute of Technology 